\title{
Activity Recognition From Smartphone Data Using WSVM-HMM Classification
}

\author{
M'hamed Bilal Abidine, Electronics and Computer Sciences Department, University of Science and Technology Houari \\ Boumediene (USTHB), Algeria \\ Belkacem Fergani, Electronics and Computer Sciences Department, University of Science and Technology Houari \\ Boumediene (USTHB), Algeria
}

\begin{abstract}
A lot of real-life mobile sensing applications are becoming available nowadays. The traditional approach for activity recognition employs machine learning algorithms to learn from collected data from smartphone and induce a model. The model generation is usually performed offline on a server system and later deployed to the phone for activity recognition. In this paper, the authors propose a new hybrid classification model to perform automatic recognition of activities using built-in embedded sensors present in smartphones. The proposed method uses a trick to classify the ongoing activity by combining weighted support vector machines (WSVM) model and hidden Markov model (HMM). The sensory inputs to the classifier are reduced with the linear discriminant analysis (LDA). They demonstrate how to train the hybrid approach in this setting, introduce an adaptive regularization parameter for WSVM approach, and illustrate how the proposed method outperforms the state-ofthe-art on a large benchmark dataset.
\end{abstract}

\section{KEYWORDS}

Activity Recognition, HMM, Hybrid Classification, Smartphone Data, WSVM

\section{INTRODUCTION}

Embedded Sensors are ubiquitous and are becoming sophisticated by nature. This has been changing people's daily life and has opened the doors for many interesting data mining applications. Human activity recognition (HAR) is a research domain behind many applications on smartphone such as health monitoring, fall detection, context-aware mobile applications, human survey system, home automation, etc. The HAR systems consist to identify the actions being carried out by a person given a set of observations of him/herself and the surrounding environment. Recognition can be accomplished by exploiting the information retrieved from various sources such as environmental sensors (Fahim, et al., 2013), body-worn sensors (Helbostad, et al., 2017; Liang et al., 2018) or the smartphone sensors (Shoaib et al., 2015; Siirtola et al., 2012; Bayat et al., 2014).

Recent developments in sensing technology have led to wireless sensor networks which provide a non-intrusive, privacy friendly and easy to install solution to in-home monitoring (Van Kasteren et al., 2010). Sensors used are generally contact switches to measure open-close states of doors and cupboards; pressure mats to measure sitting on couch or lying in bed; mercury contacts for movement of objects such as drawers; passive infrared sensors to detect motion in a specific area and float sensors to measure the toilet being flushed. 
Automatic activity recognition systems aim to capture the state of the user and its environment by exploiting heterogeneous sensors attached to the subject's body.The such systems can monitor and keep track of the activities of daily living (ADL), learn from them and assist us in making decisions. Such assistive technologies can be of immense use for remote health care (Manirabona et al., 2018; Nimkar et al., 2019), for elderly people, the disabled, and those with special needs.

Human physical activities range from simple full body motor activities like walking, sitting and standing to complex motor activities such as jogging and climbing, the recognition of which plays an important role in many applications such as human-computer interaction and surveillance (Anguita et al., 2013; Liang et al., 2018). Performance in these activities can also be important indicators for patients recovering from newly acquired disability or people who are at risk of decline, due to aging factor. For e.g. a person staying away from his/her elderly parent can monitor their daily activities by providing an alarm if there is a change in the regular pattern or an early alarm of a health care emergency.

Some approaches have adapted dedicated motion sensors in different parts of the body, such as the waist, wrist, chest and thighs achieving good classification performance (Shoaib et al., 2014). These sensors are usually un-comfortable for the common user and do not provide a long-term solution for activity monitoring (e.g. sensor repositioning after dressing (Bao et al., 2004). Activity recognition using wearable body motion sensors has attracted more interests for decades (Lara et al., 2013).

Smartphones are becoming an integral part of daily human life (Kwapisz et al., 2011), and they are being preferred as the most usable appliances that could recognize human activities due to its powerful in terms of mobility, user-friendly interface, network capability, strong CPU, memory, and battery (Shoaib et al., 2015). They contain a large number of hardware sensors such as accelerometer, gyroscope, compass, barometer, temperature, humidity, light sensor and GPS receiver. The availability of different sensors encourages implementation of human activity recognition system and makes the smartphone a rich environment for many systems such as healthcare system.

Building a system to accurately identify these activities is a challenging task. HAR using smartphone data is a classical multivariate time series, for which the task is to detect and classify those contiguous portions of sensor data streams that cover various activities of interest. In general, most of smartphone-based HAR systems are built with four major components: sensory data acquisition, data processing and feature extraction, model training from collecting labeled data, and pattern recognition.

Sensor data can be processed in real-time or logged for offline analysis and evaluation. The model generation is usually performed offline on a server system and later deployed to the phone to recognize the activity performed.

The first component utilizes various sensors embedded in smartphones to gather data from human activities. These sensors can be used alone (Siirtola et al., 2012; Bayat et al., 2014), or combined together (Shoaib et al., 2013; Chetty et al., 2015; Capela et al., 2016) to record data generated from human activities.

The second component for HAR is an important task. It consists of transforming raw signal data into feature vectors for classification task, where a fixed length analysis window is shifted along the signal sequence for frame extraction. Statistical features such as mean, standard deviation, entropy, correlation coefficients, etc. are the most widely used handcrafted features in the HAR (Figo et al., 2010). Fourier transform and wavelet transform (Hea et al., 2009) are another two commonly used handcrafted features, while the discrete cosine transform (DCT) have also been applied with promising results (Tamura et al., 1997), as well as auto-regressive model coefficients (He, 2008). The third component is built by using different classification methods. This phase is divided into Training and Classification. In the training phase, which is conducted offline, the model is built and tuned with the optimal parameters. After constructing the optimized model, it becomes ready to use in classification phase. In this paper, we mainly focus on feature extraction, training, and classification phases infer what activity an individual is engaged in. 
The traditional approach for intelligent HAR employs machine learning algorithms to induce a model. The accuracy of the system depends on the quality of the training model. This paper mainly introduces a new hybrid classification approach that combines Weighted Support Vector Machines (WSVM) and Hidden Markov Model (HMM). We choose WSVM and HMM classifiers because they are widely used for HAR (Ordonez et al., 2013; Abidine et al., 2018). WSVM has been applied to investigate the effect of overweighting the minority class on SVM modeling between the performed activities and it deals with a "class-imbalance problem" (Abidine et al., 2018). To improve the training of HMM method, we used a new model of WSVM to estimate the probabilities of transitioning between states that represent the physical activities. This aims to overcome some of inherent limitations of the traditional HMM approach. The model generation is usually conducted offline where the model is built and fine-tuned with the optimal parameters on a server system and later transferred to the phone to infer the user's activities according to these observations. Therefore, we do not consider such growth of complexity a real problem in our work. The research may be significant in the field of pervasive healthcare, supporting a variety of practical applications such as elderly care, ambient assisted living and remote monitoring.

The first step of the proposed method is to reduce the data obtained from various sensors. There are two ways to perform the feature dimension reduction: feature extraction and feature selection (Guyon et al., 2006). In our work, we used the feature extraction approach. Feature extraction transforms the original high dimensional data into a lower dimensional feature space. The transformation can be linear or nonlinear. In this work, we employed Linear Discriminant Analysis (LDA) (Lara et al., 2013) to extract the feature vectors. The entire HAR pipeline is shown in Figure 1.

Figure 1. Human activity recognition system

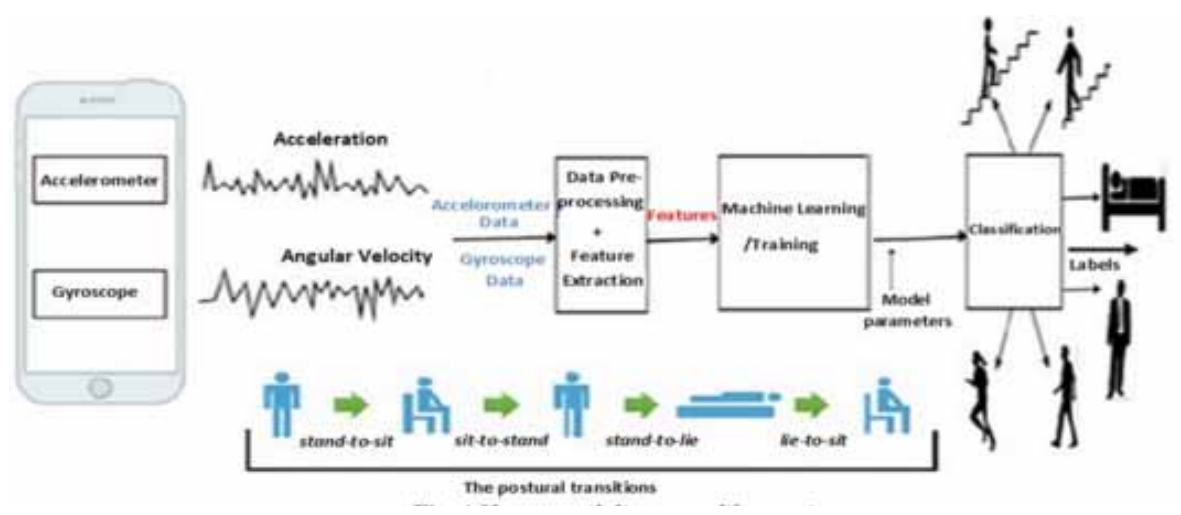

The efficiency of the proposed technique in term of high accuracy can be explained by the fact that the training phase is more robust because it was done twice using WSVM learning and HMM learning. Fortunately, the training phase in a deployed activity recognizer is usually done offline and the classification model is stored, so we do not consider such growth of complexity a real problem in our work. Another contribution of this paper is the new method to estimate the probabilities of emissions at each state for HMM learning based on the outputs of WSVM.

The remainder of the paper is organized as follows: Section 2 explains the related work and introduces a comparison between WSVM and HMM. Next, in Section 3, we used the supervised activity class prediction problem. We describe the proposed classification method WSVM-HMM. In Section 4, we give a description of the employed datasets, and describe some of the evaluation statistics of the activity recognition. Then, we discuss the experimental results obtained on real smartphone datasets. Finally, we present further analysis of our classifiers, which in turn motivate our future work. 


\section{RELATED WORKS}

There are various classifiers that have been implemented on smartphone data in the last few years such as the Support Vector Machine (SVM) (Anguita et al., 2012), Random Forest (Peterek et al., 2014), HMM (Ronao et al., 2014), k-Nearest Neighbor (kNN) (Kose et al., 2012), Decision Tree (Yan et al., 2012), Naive Bayes (Kose et al., 2012), Artificial Neural Networks (ANN) (Khan et al., 2013), Boosting algorithm (Reiss et al., 2013), Multi-layered classifiers, Rule-based classifier, Quadratic discriminant analysis, Fuzzy classification (Shoaib et al., 2015; Lara et al., 2013). Since there are many challenges in designing an HAR system, despite the amount of work that has been done in this area (Su et al., 2014; Ordóñez et al., 2012; Lester et al., 2006), the performance of HAR systems is still far from optimal.

In the activity recognition field, hybrid approaches have been successfully employed. For example, in (Ordóñez et al., 2012), the authors showed that an ANN could be hybridized with HMMs to deal with the activity recognition problem. Lester et al. developed a hybrid model that combined a modified version of AdaBoost with HMMs, and demonstrated it to be quite effective in recognizing various human activities using wearable devices. A decision tree and a Dynamic Hidden Markov Model (DHMM) are used in combination in (Walse et al., 2016). Anguita et al. in (Anguita et al., 2013) proposed the Multiclass Support Vector Machine approach (MC-SVM), where the One-Vs-All (OVA) approach is used because it learned model uses less memory when compared to the OneVs-One (OVO) method. This is advantageous for systems having few resources. In (Anguita et al., 2012) the authors introduced the concept of a Multi Class Hardware-Friendly SVM (MC-HF-SVM). This method was designed for binary classification problems by employing fixed point arithmetic (number of bits) in the feed-forward phase of the SVM classifier, with the purpose of allowing its use for battery-constrained devices. The authors state that the proposed method improves in terms of computational cost while maintaining similar accuracy when compared to traditional SVM. In (Menhour et al., 2018), the authors developed new schemes named PCA/KNN-SVM and LDA/ KNN-SVM and demonstrated that LDA has shown to be more effective than PCA due to separability criteria between classes in a high-dimensional implicit feature space.

In the Table 1, we show the advantages and disadvantages of the WSVM and HMM methods. Merging two types of classification techniques would give complementary decisions and advance the accuracy level as in the results of hybrid approach HMM/ANN that obtains a significant and notable better performance in (Ordóñez et al., 2012). We consider that WSVM based approaches have great potential and further uses in this human activity recognition problem.

\section{WSVM-HMM SYSTEM BASED LDA FEATURES}

One significant drawback in WSVMs is that, they are inherently static classifiers - they do not implicitly model temporal evolution of data. HMM has the advantage of being able to handle dynamic data with certain assumptions about stationary and independence. Taking advantage of the relative strengths of these two classification paradigms, we have developed a hybrid WSVM-HMM architecture using our training method to increase recognition performance. Figure 2 shows the architecture of the proposed activity recognition system combining WSVM and HMM. Sensor data for different activities has been collected from multiple sensors on smartphone simultaneously. Data has also been divided into two partitions: training data and testing data. First, we reduce the number of features by the LDA method in order to obtain the best discrimination between the classes in the new LDA space. Second, we train and test the Weighted SVM on the LDA features to generate an estimate of the label vector of the predicted classes $\tilde{y}_{W S V M}=\left[\begin{array}{llll}\tilde{y}_{1} & \tilde{y}_{2} & \cdots & \tilde{y}_{m}\end{array}\right]$. The final classification is performed by the 'Viterbi' algorithm using the LDA features, by the use of an HMM model. In this work, we used a new approach to estimate the emission model of HMM. This emission model is calculated from the 
Table 1. Advantage and Disadvantages of WSVM and HMM Algorithms

\begin{tabular}{|c|c|c|}
\hline Algorithms & Advantages & Disadvantages \\
\hline $\begin{array}{l}\text { Weighted Support Vector } \\
\text { Machines } \\
\text { (WSVM) }\end{array}$ & $\begin{array}{l}\text { - Faster inference } \\
\text {-Less over fitting, robust to noise } \\
\text { - Performs linear and nonlinear } \\
\text { Classification problems } \\
\text { - Adapted for the imbalanced dataset }\end{array}$ & $\begin{array}{l}\text { - Slow training } \\
\text {-SVM is a binary classifier; to do a multi-class } \\
\text { classification, pair wise classifications are used } \\
\text { - Sensitive to lots of irrelevant attributes } \\
\text { - Sensitive to outliers in input space }\end{array}$ \\
\hline $\begin{array}{l}\text { Hidden Markov Model } \\
\text { (HMM) }\end{array}$ & $\begin{array}{l}\text { - Fast training } \\
\text {-Efficient learning algorithms- learning } \\
\text { can take place directly from raw } \\
\text { sequence data } \\
\text { - The creation of the HMM model is } \\
\text { simple and fast. } \\
\text { - An update of the model is possible } \\
\text { with the algorithm of Baum-Welch } \\
\text { - Used to model complex activities. }\end{array}$ & $\begin{array}{c}\text {-Incapable of dealing the imbalanced problem } \\
\text { - Cannot express dependencies to its } \\
\text { assumptions between hidden states } \\
\text { - No way to configure the classifier } \\
\text { - Observed values must be whole numbers } \\
\text { - The prediction of a hidden state is only made } \\
\text { with a single observed value. }\end{array}$ \\
\hline
\end{tabular}

Figure 2. Hybrid WSVM-HMM recognition approach

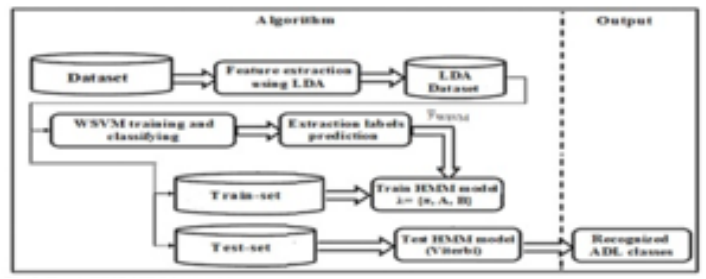

estimated label vector $\tilde{y}_{W S V M}$ and the original label vector of dataset (see Section 3.3). An estimated label vector is generated by the 'Viterbi' algorithm and the system will output the recognized activity.

Feature extraction by Linear Discriminant Analysis (LDA)

LDA seeks directions that are efficient for discrimination (Wu et al., 2010). We first define Between-Class Scatter matrix $S_{B}$ and Within-Class Scatter $S_{w}$ by

$S_{B}=\sum_{i=1}^{N} N_{i}\left(\bar{m}_{i}-\bar{m}\right)\left(\bar{m}_{i}-\bar{m}\right)^{T}$

$S_{w}=\sum_{i=1}^{N} \sum_{j=1}^{N_{i}}\left(x_{j}-\bar{m}_{i}\right)\left(x_{j}-\bar{m}_{i}\right)^{T}$

Where, $N$ is the number of activity class, $\bar{m}_{i}$ is the average value of the samples in class $\mathrm{C}_{\mathrm{i}}$. The average of the total samples is $\bar{m} . \mathrm{N}_{\mathrm{i}}$ is the number of data samples in class $\mathrm{C}_{\mathrm{i}}$. The steps of the algorithm are as follows

1. Compute the total mean vector: 


$$
\bar{m}=\frac{\sum_{i=1}^{N} x_{i}}{N}
$$

2. Compute the mean vector for each class

$$
\bar{m}_{i}=\frac{\sum_{i=1}^{N i} x_{i}}{N_{i}}
$$

3. Compute the between-class scatter: $S_{B}$

4. Compute the within-class scatter: $S_{w}$

5. Solves the generalized eigenvalue problem for the matrix $S_{w}^{-1} S_{B}$

$$
[V, D]=\operatorname{eig}\left(S_{w}^{-1} S_{B}\right)
$$

6. Get the projection matrix: $\mathrm{M}$ is composed by the top $n$ eigenvectors corresponding to the largest eigenvalues.

\section{Weighted Support Vector Machines (WSVM)}

Weighted SVM (Osuna et al., 1997; Tian et al., 2011; Huang et al., 2005) implements cost sensitive learning for SVM modeling. It also reduces the effect of imbalance data and is a promising candidate for addressing the rare class problem. WSVM in its basic form corresponds to a two class classification problem. We consider $E=\left\{\left(x_{i}, y_{i}\right), x_{i} \in R^{n}, y_{i} \in\{+1,-1\}, i=1\right.$ to $\left.m\right\}$ the training dataset where the input $x_{i}$ corresponding to the $(n)$ feature vector, obtained using the LDA transform, is labelled either belonging to the class $\left(y_{i}=+1\right)$ or not $\left(y_{i}=-1\right)$. The aim of SVM is to find the maximum-margin hyperplane in the feature space that best separates the points $y_{i}$ whose associated $y_{i}=+1$ from those points whose $y_{i}=-1$. The feature space is built through some non-linear mapping $\Phi$ of $x_{i}$ whose exact knowledge is not necessarily known, and one often limits to the knowledge of the associated 'Kernel Function', which satisfies the Mercer's condition (Huang, 2005). A typical kernel function commonly employed in this context is the Gaussian radial basis function as follows:

$$
K\left(x_{1}, x_{2}\right)=\exp \left(-\left\|x_{1}-x_{2}\right\|^{2} / 2 \sigma^{2}\right)
$$

Osuna et al (Osuna et al., 1997) proposed a Weighted SVM to deal with the imbalanced dataset by introducing two different cost misclassification parameter $C_{-}$and $C_{+}$in SVM optimization primal problem for the minority and majority classes, respectively, as given in the primal Lagrangian Eq.(7) below. Using $m_{+}$(resp. $m_{-}$) the number of positive (resp. negative) instances in the dataset ( $\left.m_{-}+m_{+}=m\right)$. We assume positive class to be the majority class and negative class to be the minority class.

$$
L_{p}=\frac{1}{2}\|w\|^{2}+C_{+} \sum_{i \mid d_{i}=1}^{m_{+}} \zeta_{i}+C_{-} \sum_{i \mid d_{i}=-1}^{m_{-}} \zeta_{i}-\sum_{i=1}^{m} \alpha_{i}\left[y_{i}\left(w \cdot x_{i}+b\right)-1+\zeta_{i}\right]-\sum_{i=1}^{m} r_{i} \zeta_{i}
$$

Subject to $\sum_{i=1}^{m} \alpha_{i} y_{i}=0,0 \leq \alpha_{i}^{+} \leq C_{+}$, and $0 \leq \alpha_{i}^{-} \leq C_{-}, i=1, \ldots, m$ 
Where $\alpha_{i}^{+}$and $\alpha_{i}^{-}$represent the Lagrangian multipliers of positive and negative examples, respectively. The $w$ parameter stands for the weight vector normal to the hyperplane that will be determined as part of the optimization problem (7). The WSVM method introduces non-negative slack variables $\zeta_{i}$, which measure the degree of misclassification of $x_{i}$.

The dual optimization problem of WSVM with different constraints on $\alpha_{i}$ can be solved similarly to (Huang et al., 2005):

$$
\max _{\alpha_{i}}\left\{\sum_{i=1}^{m} \alpha_{i}-\frac{1}{2} \sum_{i=1}^{m} \sum_{j=1}^{m} \alpha_{i} \alpha_{j} y_{i} y_{j} K\left(x_{i}, x_{j}\right)\right\}
$$

Solving the dual optimization problem of WSVM gives a decision function in the original space for classifying a test point $y \in R^{n}$ with $m_{s v}$ is the number of support vectors $x_{i} \in R^{n}$.

$$
\tilde{y}_{W S V M}=\operatorname{sgn}\left(\sum_{i=1}^{m_{s v}} \alpha_{i} y_{i} K\left(x, x_{i}\right)+b\right)
$$

Three parameters can affect the decision outcome: $\mathrm{b}, \alpha_{i}$, and $\mathrm{K}$. The equality constraint (8) indicates that if there are more positive samples than negative samples, i.e. if more $\mathrm{y}_{i}$ equal +1 than -1 , then the negative class will have higher $\alpha_{i}$ values in order to guarantee a zero sum. In the decision function (9), $\alpha_{i}$ can be regarded as the weight of each example; thus larger $\alpha_{i}$ values essentially increase the influence of the minority class, which automatically rebalances the skewed dataset.

When the dataset is imbalanced, the density of majority class examples would be higher than the density of minority class examples even around the class boundary region, where the ideal hyperplane would pass through. As a consequence, in order to reduce the total number of misclassifications in SVM learning, the predicted decision boundary can be shifted (or skewed) towards the minority class, see Figure3. This shift can cause the generation of more false negative predictions, which lowers the model's performance on the minority negative class. When the class imbalance is extreme, the SVM could produce models having largely skewed hyperplanes, which would even recognize all the examples as positives. This explains why SVM fails completely in situations with a high degree of imbalance dataset, which motivates the so called Weighted Support Vector Machines (WSVM) detailed later on.

If the training data gets more imbalanced, the ratio between the positive and negative support vectors also becomes more imbalanced. Some authors (Abidine et al., 2018; Fernández Hilario et al., 2018; Yang et al., 2007), have proposed adjusting different cost parameters to solve the imbalanced problem as follows:

$$
\frac{C_{+}}{C_{-}}=\frac{m_{-}}{m_{+}}
$$

One way to deal with this problem is to increase the tradeoff $\mathrm{C}+$ associated with the positive instances as in (Abidine et al., 2018) where different misclassification $C_{i}$ per class were used to solve this problem. Especially, by taking $C_{-}=C_{i}$ and $C_{+=} C$, where $m_{+}$and $m_{i}$ stand for the number of samples of majority classes and the number of samples in the $i^{\text {th }}$ class, respectively. 
Figure 3. Weighted SVM classification problem.

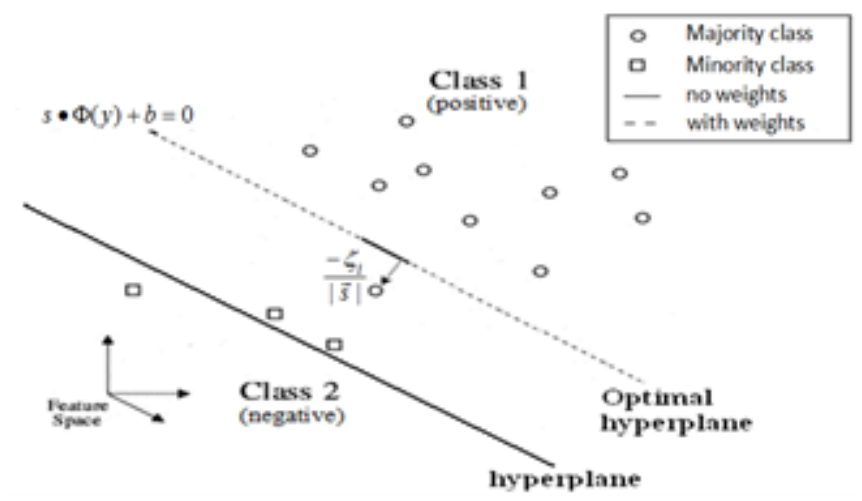

$C_{i}=\operatorname{round}\left(C \times\left[m_{+} / m_{i}\right]\right), i=1, \ldots, N$.

Where $C$ is the common cost parameter of the WSVM.

\section{Hidden Markov Model (HMM)}

The Hidden Markov Model (HMM) (Nguyen et al., 2015) is a classic way of modeling generative sequence process probabilistically, which generates hidden states $y$ from observable data $x$. It constructs the activity model by observing the effects of an activity. In our case, the hidden variable is the activity that the subject was performing at a given time step and the observed variable is the vector of sensor readings.

HMM model mainly works on two basic principles as follows:

- The observable variable at time $t$, namely $x_{\mathrm{t}}$, depends only on the hidden variable $y_{\mathrm{t}}$.

- The hidden variable at time $t$, namely $y_{t}$, depends only on the previous hidden variable $y_{t-1}$.

With these assumptions we can specify an HMM using three probability distributions: the distribution over initial states $p\left(y_{1}\right)$; the transition distribution $p\left(y_{t} \mid y_{t-1}\right)$ represents the probability of going from one state to the next; and the emission distribution $p\left(x_{t} \mid y_{t}\right)$ indicating the probability that the state $y_{t}$ would generate observation vector $x_{t}$. Learning the parameters of these distributions correspond to maximizing the joint probability $\mathrm{p}(\mathrm{x}, \mathrm{y})$ between the sensor data and activities in the training data. It is described above as follows:

$$
P(\mathrm{x}, \mathrm{y})=\prod_{t=1}^{T} p\left(y_{t} \mid y_{t-1}\right) p\left(x_{t} \mid y_{t}\right)
$$

in which we write the distribution over initial states $p\left(\mathrm{y}_{1}\right)$ as $p\left(\mathrm{y}_{1} \mathrm{l} \mathrm{y}_{0}\right)$, to simplify notation.

A HMM is quantitatively described by these parameters: $\lambda=\{\pi, A, B\}$ the distribution over initial states parameterized by $\pi=\left\{\pi_{i}\right\}$; the transition probability distribution parameterized by $A$; and the emission distribution parameterized by $B$.

$$
A=\left[a_{i j}\right]
$$


Figure 4. The graphical representation of a HMM. The shaded nodes represent observable variables, while the white nodes represent hidden ones

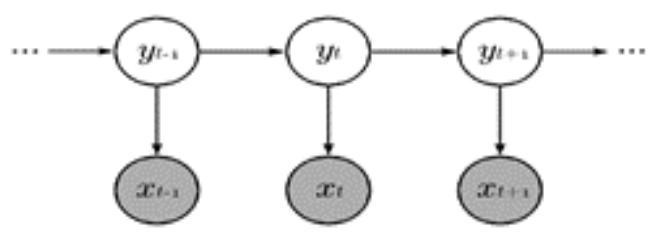

$a_{i j}=P\left(y_{t}=j \mid y_{t-1}=i\right)$, and $\sum_{j=1}^{N} a_{i j}=1$

$B=\left[b_{j}\left(O_{t}\right)\right]$

$b_{j}\left(O_{t}\right)=P\left(q_{k+1}=O_{t} / q_{t}=i\right)$

$\pi=\left[\pi_{1}, \pi_{2} \ldots, \pi_{N}\right]$

$\pi_{\mathrm{i}}=P\left(q_{0}=i\right)$

With $: i, j \in\{1,2, \ldots, N\}$

$t \in\{1,2, \ldots, T\}$

$O_{t}:$ Vector of observations

The initial state distribution $\mathrm{p}\left(\mathrm{y}_{1}\right)$ is a probability table with individual values as follows:

$p\left(y_{1}=i\right)=\pi_{i}, \pi_{i} \geq 0 \quad 1 \leq i \leq N$ and $\sum_{j=1}^{N} \pi_{i}=1$

In our work, for estimating the emission distribution $B=b_{j}\left(O_{t}\right)$ at each state, we consider $O_{t}=$ $\tilde{y}_{\text {WSVM }}$ will be the label vector generated by WSVM algorithm (Label_WSVM) and $\mathrm{q}_{\mathrm{t}}$ represent the vector of the original activity classes (Labels) of the dataset. The pseudo code for constructing the matrix of emission $B$ is outlined as follows.

HMM used the Baum-Welch supervised learning algorithm to estimate the transition probabilities between states and the Viterbi algorithm (Cheng et al., 2010) to predict the series of states for a test sequence given emissions. The pseudo-code in Table 3 illustrates the execution of the suggested multiclass LDA/WSVM-HMM

\section{EXPERIMENTAL RESULTS AND DISCUSSION}

In this section, we give a description of the employed datasets, then the testing setup is detailed, and finally the results are highlighted and discussed. 
Table 2. Construction of matrix B for HMM learning

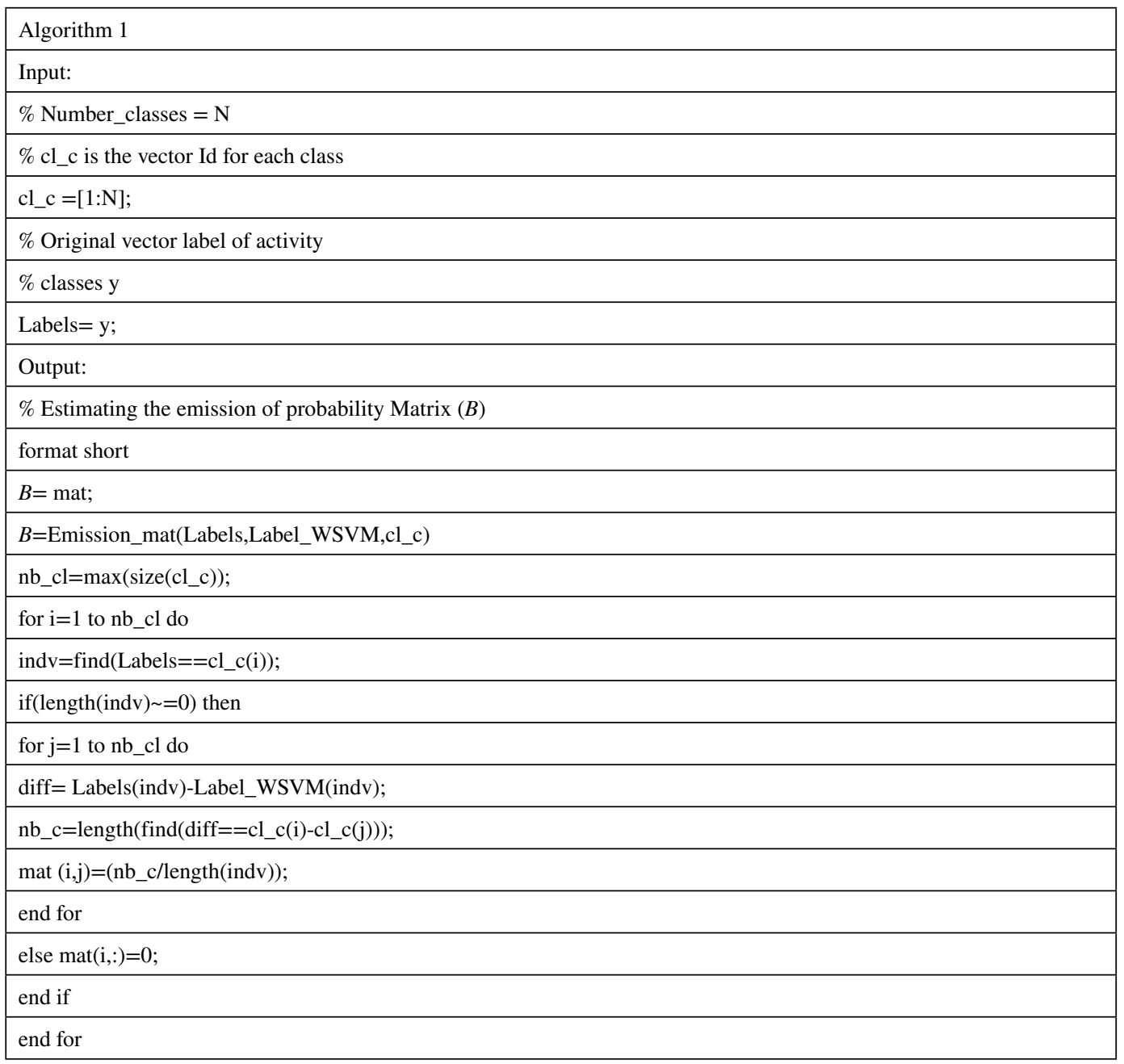

\section{Datasets Description}

In this paper, we use four publicly available online datasets. The first dataset used is from Human activity recognition (Dataset 1, 2017). The second dataset (HAPT) with Postural Transitions (Dataset2, 2016) is quite similar to the previous one, but, it includes postural transitions. The third dataset is from (Dataset3, 2017), is known as the Sensors Activity Recognition (SAR). The last dataset is from (Dataset4, 2017), titled Wireless Sensor Data Mining (WISDM 2017). All four datasets have been recorded by means of Android smartphones and their characteristics, in order to ease the comparison. These datasets vary in their formats, types of sensors they are generated from, and sampling frequencies. Some of these datasets included 3 axis gyroscope measurements, in addition to the 3 axis accelerometer measurements common to all, with the exception of the WISDM Lab 
Table 3. Summary of the proposed method for activity recognition

\begin{tabular}{|c|c|}
\hline \multicolumn{2}{|l|}{ Algorithm 2} \\
\hline \multicolumn{2}{|l|}{ Input: Raw data (Features, Labels) } \\
\hline \multicolumn{2}{|l|}{ Output: Viterbi Predict labels } \\
\hline \multicolumn{2}{|l|}{ Data features $\neg$ Raw data. Features; } \\
\hline \multicolumn{2}{|l|}{ Data labels $\neg$ Raw data. Labels; } \\
\hline \multicolumn{2}{|l|}{ // Feature extraction using LDA algorithm (step 1) } \\
\hline \multicolumn{2}{|l|}{ for ith_partition $=1$ to 10 do } \\
\hline \multicolumn{2}{|c|}{$\begin{array}{l}\text { LDA Train Parameters } \neg \text { LDA Train Algorithm (Data features [without ith_partition], Data labels [without ith_ } \\
\text { partition]); }\end{array}$} \\
\hline \multicolumn{2}{|c|}{ LDA Features [ith_partition] $\neg$ LDA Features Algorithm (Data features [ith_partition], LDA Train Parameters); } \\
\hline \multicolumn{2}{|c|}{ end for } \\
\hline \multicolumn{2}{|l|}{$/ / 1^{\text {st }}$ classification using WSVM (supervised method) (step 2) } \\
\hline \multicolumn{2}{|l|}{ for ith_partition $=1$ to 10 do } \\
\hline \multicolumn{2}{|l|}{ WSVM Train Model [without ith_partition] $\neg$} \\
\hline \multicolumn{2}{|l|}{ WSVM Train Algorithm (LDA Features [without ith_partition], Data labels [without ith_partition]); } \\
\hline \multicolumn{2}{|l|}{ WSVM Predict labels [ith_partition] $\neg$} \\
\hline \multicolumn{2}{|l|}{ WSVM Test Algorithm (WSVM Train Model [without ith_partition], LDA Features [ith_partition]); } \\
\hline \multicolumn{2}{|l|}{ end for } \\
\hline \multicolumn{2}{|l|}{$/ / 2^{\text {nd }}$ classification using Viterbi algorithm avec HMM model (supervised method) (step 3) } \\
\hline \multicolumn{2}{|l|}{ for ith_partition $=1$ to 10 do } \\
\hline \multicolumn{2}{|l|}{ HMM Train Model [without ith_partition] $\neg$} \\
\hline \multicolumn{2}{|l|}{ HMM_Training_Algorithm (WSVM_Label [without ith_partition], Original_Label [without ith_partition]); } \\
\hline \multicolumn{2}{|l|}{ Viterbi Predict labels [ith_partition] $\neg$} \\
\hline \multicolumn{2}{|l|}{ Viterbi_Algorithm (HMM Train Model [without ith_partition], WSVM_Label [ith_partition]); } \\
\hline end for & \\
\hline
\end{tabular}

public dataset which used tri-axial accelerometer data. The HAR and HAPT datasets provide a large number of features (see the Table 4) extracted by prepossessing the raw signals generated from the accelerometer and the gyroscope sensors. For the annotation of the activities, the video-recording is used to label the data manually.

As mentioned previously, Sensor data streams were segmented in time slices of constant length fixed in our experiments. Table 5 enables us to visualize the disparity between activities in terms of number of observations (particularly for the HAPT dataset, e.g. Walking and Sit to Stand). Therefore, the HAPT dataset suffers from a severe class imbalance due to the nature of the data.

\section{Data Analysis Setup}

To evaluate the performance of classifiers, in particularly for the rare class, we use F-measure, which is defined as the harmonic mean of recall and precision in the table 6 . Precision measures the percentage of activities inferred activities correctly recognized while recall measures the percentage of ground truth activities correctly recognized. They can be calculated by Eq. (20), (21), (22), and (23) respectively. As for the multi classification issue, the example (data with feature and label) can be divided into four categories according to classification result and ground truth: $T P_{i}$ (true positive) 
Table 4. Summary of datasets used in evaluation of the proposed approach. Accelerometer (A), Gyroscope (G), Magnetometer (M)

\begin{tabular}{|c|c|c|c|c|}
\hline Houses & HAR & HAPT & SAR & WISDM \\
\hline Age (years) & [19-48] & [19-48] & {$[25-30]$} & - \\
\hline $\mathrm{Nb}$ of subjects & 30 & 30 & 10 & 29 \\
\hline Annotation & Video & Video & PDA & $\begin{array}{l}\text { Graphical user } \\
\text { interface }\end{array}$ \\
\hline Features & 561 & 561 & 9 & 6 \\
\hline Smartphone & Samsung Galaxy SII & Samsung Galaxy SII & $\begin{array}{l}\text { Samsung } \\
\text { GalaxySII }\end{array}$ & Cell Phone \\
\hline Position & Waist & Waist & Belt & Front leg pocket \\
\hline Sensors & $A$ and $G$ & $A$ and $G$ & $\mathrm{~A}, \mathrm{G}$ and $\mathrm{M}$ & A \\
\hline Activities & 6 & 12 & 6 & 6 \\
\hline
\end{tabular}

Table 5. Annotated list of physical activities.

\begin{tabular}{|c|c|c|c|c|c|}
\hline Activities & Status & HAR & HAPT & SAR & WISDM \\
\hline Walking & Dynamic & 1012 & 1722 & 31751 & 2081 \\
\hline $\begin{array}{c}\text { Walking_ } \\
\text { upstairs }\end{array}$ & Dynamic & 858 & 1544 & 21903 & 632 \\
\hline $\begin{array}{c}\text { Walking_ } \\
\text { downstairs }\end{array}$ & Dynamic & 930 & 1407 & 18751 & 528 \\
\hline Sitting & Static & 1123 & 1801 & 30000 & 306 \\
\hline Standing & Static & 1029 & 1979 & 30000 & 246 \\
\hline Laying & Static & 792 & 1958 & - & - \\
\hline Jogging & Dynamic & - & - & 29402 & 1625 \\
\hline Stand to Sit & Transition & - & 70 & - & - \\
\hline Sit to Stand & Transition & - & 33 & - & - \\
\hline Sit to Lie & Transition & - & 107 & - & - \\
\hline Lie to Sit & Transition & - & 85 & - & - \\
\hline Stand to Lie & Transition & - & 139 & - & - \\
\hline Lie to Stand & Transition & - & 84 & - & - \\
\hline
\end{tabular}

Table 6. The Confusion matrix. The $\epsilon_{i j}$ terms show the error between true class $i$ and inferred class $j$.

\begin{tabular}{|c|c|c|c|c|}
\hline & \multicolumn{3}{|c|}{ Inferred } & FN \\
\hline True & 1 & 2 & 3 & $F N_{1}$ \\
\hline 1 & $T P_{1}$ & $\epsilon_{12}$ & $\epsilon_{13}$ & $F N_{2}$ \\
\hline 2 & $\epsilon_{21}$ & $T P_{2}$ & $\epsilon_{23}$ & $F N_{3}$ \\
\hline 3 & $\epsilon_{31}$ & $\epsilon_{32}$ & $T P_{3}$ & Total \\
\hline FP & $F P_{1}$ & $F P_{2}$ & $F P_{3}$ & \\
\hline
\end{tabular}


is the number of true positives for an activity $i, F P_{i}$ (false positive) is the number of false positives for activity $i, T N_{i}$ is the number of true negatives for an activity $i$ and $F N_{i}$ (false negative) is the number of false negatives for activity $i$. The confusion matrix is shown in the following table.

These measures are calculated as follows:

Accuracy $=\frac{\sum_{i=1}^{N} T P_{i}}{\text { Total }} \times 100 \%$

Precision $=\frac{1}{N} \sum_{i=1}^{N}\left[\frac{\mathrm{TP}_{i}}{\mathrm{TP}_{i}+\mathrm{FP}_{i}}\right] \times 100 \%$

Recall $=\frac{1}{N} \sum_{i=1}^{N} \frac{\mathrm{TP}_{i}}{\mathrm{TP}_{i}+\mathrm{FN}_{i}} \times 100 \%$

$\mathrm{F}-$ score $=\frac{\left(1+2^{2}\right) \cdot \text { Precision } \cdot \text { Recall }}{{ }^{2}{ }^{2} \cdot \text { Precision }+ \text { Recall }} \times 100 \%$

F-score and accuracy return values between $[0,100]$, where a value near to 100 shows the best performance, and near to 0 indicates the worst performance. In an extremely imbalanced dataset, the overall classification accuracy is considered not an appropriate measure of performance, but this measure was used to evaluate the accuracy of each activity class.

\section{Results}

Feature extraction stage: In this study, an approach based on LDA algorithm is used to select the best extracted features from the segmented raw data. See Figure 5 for the feature process. The input dimensionality is reduced by selecting the number of extracted features that directly equal to $\mathrm{N}-1$ dimensional feature space, where $N$ is the number of class activities.

Classification stage: These algorithms are tested under MATLAB environment and the Weighted SVM algorithm is tested with an implementation from LibSVM (Hsu et al., 2011) with Gaussian kernel is used for all the datasets. Each training dataset is normalized before classification within a range of $[-1,1]$.

To study the effects of different $\sigma$ on the performance of WSVM when the RBF kernel function is used, we conduct several experiments using different SVMs and hyper-parameter $\sigma$ in the range $[0.1,0.2,0.5,1,2,3]$ to maximize the error rate of 5- fold- cross validation technique. We found the optimal values $\sigma_{\text {opt }}=0.9, \sigma_{\text {opt }}=0.9, \sigma_{\text {opt }}=1$ and $\sigma_{\text {opt }}=0.8$ for the training dataset of HAR, HAPT, SAR, and WISDM respectively. For the WSVM classification, a local optimization was done to the cost parameter $C_{i}$ adapting to different classes where the common cost parameter $C$ is fixed to 0.1 .

Table 7 depicts the overall performance of our approach in terms of Accuracy, Recall, Precision, and F-score, during the testing phase. Our results using the proposed method show a remarkable performance in terms of F-score on all datasets, compared to other baseline methods (Anguita et al., 2013; Anguita et al., 2012; Fu et al., 2014; Zainudin et al., 2017; Kwapisz et al., 2011), by recognizing different activities perfectly. For instance, with HAR dataset, we obtained a classification error of $3.9 \%$ 

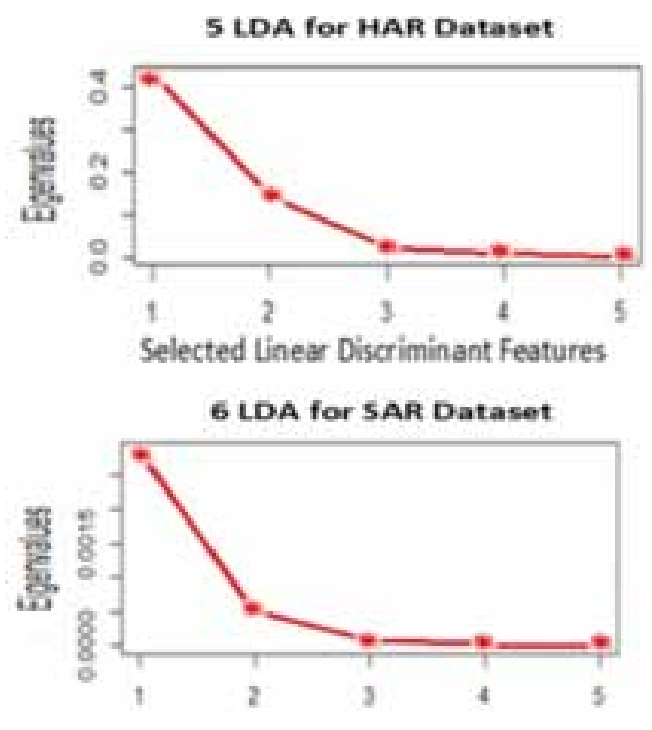

Selected Linear Discriminant Features

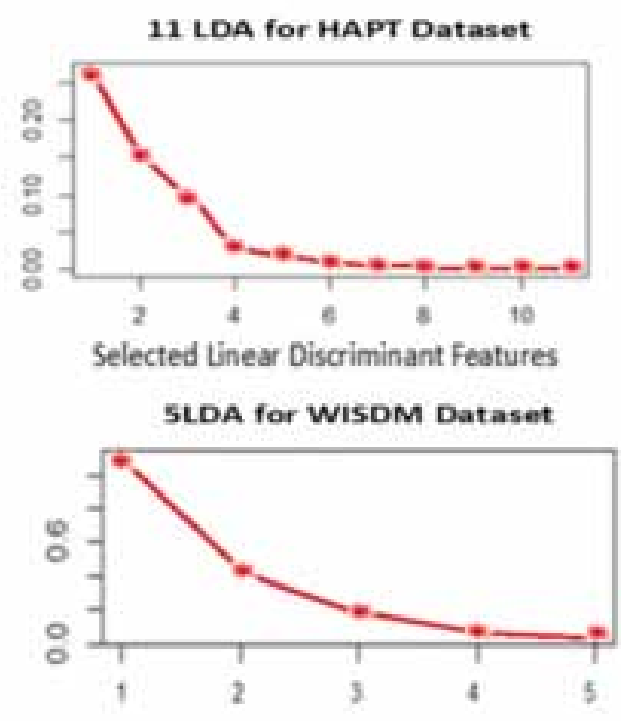

Selected tinear Discriminant Features

in terms of F-score on the LDA/WSVM-HMM method; it shows a decrease of the error comparatively to other methods. The MC-SVM and MC-HF-SVM methods present more than $10 \%$ of error.

On the other hand, the results also show that WSVM outperforms HMM for recognizing activities for all datasets except for the HAPT dataset. This can be explained by the fact that HAPT dataset contains the transition activities and HMM makes the transition states as activities: e.g. Sit-Stand. One also notices that the combined methods LDA-WSVM and LDA-HMM improve the classification results over WSVM and HMM classifiers used alone.

\section{Discussion}

To get a detailed knowledge of the performances on each class corresponding to current activity, we calculate the confusion matrix of the proposed method in Tables 8, 9 for the HAR and WISDM datasets respectively with six different activities. From these tables, we see that the performance was greatly affected by the data imbalance problem as in the WISDM dataset, in particular for the dynamic activities. 
Table 7. Comparison of existing approaches with the proposed approach (LDA/WSVM-HMM).

\begin{tabular}{|c|c|c|c|c|c|}
\hline Datasets & Approach & Accuracy (\%) & Recall (\%) & Precision (\%) & F-score $(\%)$ \\
\hline HAR & $\begin{array}{l}\text { MC-SVM (Anguita } \text { et al., } \\
\text { 2012) } \\
\text { MC-HF-SVM (Anguita et } \\
\text { al., 2012) } \\
\text { Decision Trees (Bharathi } \text { et } \\
\text { al., 2020) } \\
\text { MLP (Bharathi } \text { et al., 2020) } \\
\text { Random Forest (Bharathi et } \\
\text { al., 2020) } \\
\text { Logistic Regression } \\
\text { (Bharathi et al., 2020) } \\
\text { LSTM (Bharathi } \text { et al., } \\
\text { 2020) } \\
\text { CNN (Bharathi et al., 2020) } \\
\text { WSVM } \\
\text { HMM } \\
\text { LDA-WSVM } \\
\text { LDA-HMM } \\
\text { Proposed }\end{array}$ & $\begin{array}{l}89.3 \\
89.0 \\
82.4 \\
98.4 \\
89.5 \\
96.1 \\
94.5 \\
94.1 \\
96.4 \\
93.9 \\
96.2 \\
94.7 \\
96.7\end{array}$ & $\begin{array}{l}89.6 \\
89.3 \\
- \\
\\
95.1 \\
89.1 \\
96.1 \\
\\
94.5 \\
91.9 \\
91.5 \\
89.2 \\
93.4 \\
91.1 \\
\mathbf{9 4 . 5}\end{array}$ & $\begin{array}{l}89.9 \\
89.2 \\
- \\
\\
95.6 \\
89.6 \\
\\
96.4 \\
\\
94.6 \\
94.0 \\
88.7 \\
90.2 \\
89.4 \\
91.7 \\
97.9\end{array}$ & $\begin{array}{c}89.7 \\
89.2 \\
\\
82.9 \\
95.3 \\
89.3 \\
96.2 \\
94.5 \\
92.9 \\
90.1 \\
89.7 \\
92.8 \\
91.4 \\
\mathbf{9 6 . 1}\end{array}$ \\
\hline HAPT & $\begin{array}{l}\text { MC-SVM (Anguita } \text { et al., } \\
\text { 2012) } \\
\text { TASG-SVM(Zheng } \text { et al., } \\
\text { 2018) } \\
\text { RF (Zheng } \text { et al., 2018) } \\
\text { TASG-RF (Zheng } \text { et al., } \\
\text { 2018) } \\
\text { KNN (Zheng et al., 2018) } \\
\text { TASG-KNN (Zheng et al., } \\
\text { 2018) } \\
\text { RNN (Zheng } \text { et al., 2018) } \\
\text { TASG-RNN(Zheng } \text { et al., } \\
\text { 2018) } \\
\text { WSVM } \\
\text { HMM } \\
\text { LDA-WSVM } \\
\text { LDA-HMM } \\
\text { Proposed }\end{array}$ & $\begin{array}{l}96.0 \\
96.2 \\
94.3 \\
94.6 \\
90.5 \\
92.8 \\
94.5 \\
95.8 \\
\\
86.1 \\
96.5 \\
85.4 \\
94.8 \\
\mathbf{9 5 . 8}\end{array}$ & $\begin{array}{l}96.3 \\
90.7 \\
87.4 \\
89.3 \\
82.5 \\
86.3 \\
87.1 \\
90.4 \\
96.0 \\
98.3 \\
95.0 \\
97.4 \\
97.3\end{array}$ & $\begin{array}{l}96.6 \\
90.9 \\
88.3 \\
89.6 \\
83.4 \\
87.9 \\
86.0 \\
91.2 \\
92.4 \\
97.1 \\
94.5 \\
94.8 \\
\mathbf{9 8 . 8}\end{array}$ & $\begin{array}{l}96.4 \\
90.8 \\
87.8 \\
89.4 \\
82.9 \\
87.1 \\
86.5 \\
90.8 \\
94.1 \\
97.7 \\
94.7 \\
96.1 \\
\mathbf{9 8 . 0}\end{array}$ \\
\hline SAR & $\begin{array}{l}\text { Random Forest (Zainudin et } \\
\text { al., 2017) } \\
\text { WSVM } \\
\text { HMM } \\
\text { LDA-WSVM } \\
\text { LDA-HMM } \\
\text { Proposed }\end{array}$ & $\begin{array}{l}95.6 \\
95.0 \\
94.7 \\
95.9 \\
90.8 \\
96.5\end{array}$ & $\begin{array}{l}95.6 \\
90.5 \\
94.4 \\
93.8 \\
92.0 \\
\mathbf{9 5 . 9}\end{array}$ & $\begin{array}{l}95.7 \\
97.7 \\
93.0 \\
97.8 \\
97.1 \\
\mathbf{9 9 . 7}\end{array}$ & $\begin{array}{l}95.6 \\
94.0 \\
93.7 \\
95.8 \\
94.5 \\
\mathbf{9 7 . 7}\end{array}$ \\
\hline WISDM & $\begin{array}{l}\text { J48 (Kwapisz et al., 2011) } \\
\text { LogisticRegression } \\
\text { (Kwapisz et al., 2011) } \\
\text { MultilayerPerceptron } \\
\text { (Kwapisz et al., 2011) } \\
\text { WSVM } \\
\text { HMM } \\
\text { LDA-WSVM } \\
\text { LDA-HMM } \\
\text { Proposed }\end{array}$ & $\begin{array}{l}85.1 \\
78.1 \\
\\
91.7 \\
\\
81.4 \\
84.9 \\
79.5 \\
83.5 \\
\mathbf{8 4 . 9}\end{array}$ & $\begin{array}{l}81.7 \\
68.4 \\
\\
80.4 \\
\\
83.4 \\
79.4 \\
85.6 \\
83.9 \\
86.8\end{array}$ & $\begin{array}{l}82.4 \\
70.5 \\
84.6 \\
76.5 \\
80.0 \\
82.1 \\
76.2 \\
91.2\end{array}$ & $\begin{array}{r}82.0 \\
69.4 \\
\\
82.4 \\
\\
79.8 \\
79.7 \\
83.8 \\
79.8 \\
\mathbf{8 8 . 9}\end{array}$ \\
\hline
\end{tabular}


Table 8. Confusion matrix of activities for the proposed method on the HAR dataset.

\begin{tabular}{|c|c|c|c|c|c|c|}
\hline Activities & Walking & W. Upstairs & W. Downstairs & Sitting & Standing & Laying \\
\hline Walking & $\mathbf{9 7 . 9}$ & 0.7 & 1.4 & 0.0 & 0.0 & 0.0 \\
\hline Walking_upstairs & 0.2 & $\mathbf{9 9 . 1}$ & 0.7 & 0.0 & 0.0 & 0.0 \\
\hline Walking_downstairs & 1.1 & 0.2 & $\mathbf{9 8 . 7}$ & 0.0 & 0.0 & 0.0 \\
\hline Sitting & 0.0 & 0.0 & 0.0 & $\mathbf{8 2 . 1}$ & 11.0 & 6.9 \\
\hline Standing & 0.0 & 0.1 & 0.0 & 8.0 & $\mathbf{9 0 . 2}$ & 1.7 \\
\hline Laying & 0.0 & 0.0 & 0.0 & 0.6 & 0.4 & $\mathbf{9 9 . 0}$ \\
\hline
\end{tabular}

The values are percentages

In the table $8,99.1 \%$ of 'W. Upstairs' activity instances are correctly recognized, while $0.7 \%$ goes into 'W.Downstairs' and $0.2 \%$ are confused with 'Walking' activity. The similar classes such as 'Walking', 'W. Upstairs', and 'W. Downstairs' show similar trend of sharing errors among each other. The reason is the similar status of smartphone when the user does these dynamic activities. We notice that the static activities 'Sitting', 'Standing' and 'Laying' share errors among each other. $11 \%$ of 'Standing' activity instances are confused with 'Sitting' activity and $8 \%$ of 'Sitting' activity instances are confused with 'Standing' activity. Intuitively, this can be explained by the fact that the patterns in the acceleration data between these activities are somewhat similar. However, the minority class 'Laying' in terms of number of instances (792) is rather better recognized using the proposed method.

Table 9 demonstrates that in most cases, we achieve high levels of accuracy. For the two most common activities in terms of number of instances, 'Walking' and 'Jogging', we generally achieve accuracies above $97 \%$. Jogging appears easier to identify than other activities, which seems to make

Table 9. Confusion matrix of activities for the proposed method on the WISDM dataset

\begin{tabular}{|c|c|c|c|c|c|c|}
\hline Activities & Walking & W. Upstairs & W. Downstairs & Sitting & Standing & Jogging \\
\hline Walking & $\mathbf{9 7 . 7}$ & 1.4 & 0.8 & 0.0 & 0.0 & 0.1 \\
\hline Walking. Upstairs & 8.9 & $\mathbf{6 6 . 1}$ & 24.8 & 0.0 & 0.0 & 0.2 \\
\hline Walking. Downstairs & 12.9 & 15.8 & $\mathbf{6 7 . 9}$ & 0.2 & 2.5 & 0.7 \\
\hline Sitting & 2.7 & 0.0 & 0.6 & $\mathbf{9 3 . 5}$ & 3.2 & 0.0 \\
\hline Standing & 1.1 & 0.2 & 0.0 & 1.4 & $\mathbf{9 6 . 8}$ & 0.5 \\
\hline Jogging & 0.8 & 0.0 & 0.0 & 0.1 & 0.3 & $\mathbf{9 8 . 8}$ \\
\hline
\end{tabular}

sense, since jogging involves more extreme changes in acceleration. On the contrary, it appears much more difficult to identify the two stair climbing activities $(66.1 \%$ for 'W. Upstairs' and $67.9 \%$ for 'W. Downstairs'), but as we shall see shortly, that is because those two similar activities are often confused with one another. When grouping these two activities as one (activity: stairs), the system was able to recognize it with $100 \%$ accuracy. Note that there are very few instances of 'Sitting' (306) and 'Standing' (246), but we can still identify these activities quite well with the proposed method. Although some of the activities recorded reflect somewhat insufficient performance as in WISDM dataset for the 'W. Upstairs' and 'W. Downstairs' activities, we could state that our method is capable of producing a decent accuracy. 
The impact of gyroscope and the accelerometer sensors were found to be sensitive to physical positions. Indeed, the gyroscope is unable to differentiate between similar activities like 'Sitting' and 'Standing'. On the other side, the accelerometers perform badly with 'W. Upstairs' and 'W. Downstairs'. For the WISDM dataset using the accelerometer sensor, we note that the performances are decreasing comparatively to the other datasets. This is explained by the fact that only accelerometer sensor is used and therefore it is insufficient to recognize all activities. However, the fusion sensors for collecting the datasets perform better in term of prediction accuracy. They become complementary and offer the best performances.

\section{CONCLUSION AND FUTURE WORK}

In this work, we developed a novel hybrid model WSVM-HMM applied to human activity classification using data from Smartphone from various sensor positions such as pocket and belt. Extensive experimental evaluations using different publicly available databases of human activity show the proposed strategy is highly effective. This can be explained by the fact that the learning model is more accurate because it was done twice using WSVM learning and HMM learning.

The fusion of the sequential model HMM with the WSVM model gave more chance to the HMM to improve the results. WSVM investigated the effect of overweighting the minority class on SVM modeling between the performed activities. HMM is a natural solution to address the activity complexity by — capturing and smoothing information during the activity transition period. Additionally, accuracy also tends to decrease when including few informative features to classify. All the classification methods used the reduced data by the LDA as input to select minimal number of discriminative and relevant features.

For future work, we are planning to determine the most effective sensor positions for classifying various types of activities. They are very crucial for creating highly accurate smartphone based recognition system. Furthermore, another challenging is to evaluate the performance in recognizing the activity using the combinations of several sensor placements attached to the subject's body. Additionally, we also encourage other researchers to improve upon our method in different domains. 


\section{REFERENCES}

Abidine, M. B., Fergani, L., Fergani, B., \& Oussalah, M. (2018). The joint use of sequence features combination and modified weighted SVM for improving daily activity recognition. Pattern Analysis and Applications, 21(1), $119-138$.

Anguita, D. (2013). A public domain dataset for human activity recognition using smartphones 21th European Symposium on Artificial Neural Networks, Computational Intelligence and Machine Learning, ESANN.

Anguita, D., Ghio, A., Oneto, L., Parra, X., \& Reyes-Ortiz, J. L. (2012). Human Activity Recognition on Smartphones using a Multiclass Hardware-Friendly Support Vector Machine. International Workshop of Ambient Assisted Living (IWAAL 2012). doi:10.1007/978-3-642-35395-6_30

Bao, L., \& Intille, S. S. (2004). Activity recognition from user-annotated acceleration data. IEEE Pervasive Computing, 1-17.

Bayat, A., Pomplun, M., \& Tran, D. A. (2014). A Study on Human Activity Recognition Using Accelerometer Data from Smartphones. Elsevier Procedia Computer Science - MobiSPC, 1-8.

Bharathi, B., \& Bhuvana, J. (2020). Human Activity Recognition using Deep and Machine Learning Algorithms. International Journal of Innovative Technology and Exploring Engineering, 9(4).

Capela, N. A., Lemaire, E. D., Baddour, N., Rudolf, M., Goljar, N., \& Burger, H. (2016). Evaluation of a smartphone human activity recognition application with able-bodied and stroke participants. Journal of Neuroengineering and Rehabilitation, 13(5), 1-10. doi:10.1186/s12984-016-0114-0 PMID:26792670

Cheng, B. C., Tsai, Y. A., Liao, G. T., \& Byeon, E. S. (2010). HMM machine learning and inference for Activities of Daily Living recognition. The Journal of Supercomputing, 54(1), 29-42. doi:10.1007/s11227-009-0335-0

Chetty, G., White, M., \& Akther, F. (2015). Smart Phone Based Data Mining For Human Activity Recognition. Elsevier Procedia Computer Science - ICICT, 46, 1181-1187.

Dataset1. (2017). Available: https://archive.ics.uci.edu/ml/machine-learning-databases/00240/

Dataset2. (2016). https://archive.ics.uci.edu/ml/datasets/Smartphone-Based+Recognition+of+Human+Activi ties+and+Postural+Transitions

Dataset3. (2017). http://ps.ewi.utwente.nl/Datasets.php

Dataset4. (2017). https://www.cis.fordham.edu/wisdm/dataset.php

Fahim, M., Fatima, I., Lee, S., \& Park, Y. T. (2013). Efm: Evolutionary fuzzy model for dynamic activities recognition using a smartphone accelerometer. Applied Intelligence, 39(3), 1-14. doi:10.1007/s10489-013-0427-7

Fernández Hilario, García López, Galar, Prati, Krawczyk, \& Herrera. (2018). Learning from Imbalanced Data Sets. Springer. doi:10.1007/978-3-319-98074-4

Figo, D., Diniz, P. C., Ferreira, D. R., \& Cardoso, J. M. (2010). Preprocessing techniques for context recognition from accelerometer data. Personal and Ubiquitous Computing, 14(7), 645-662. doi:10.1007/s00779-010-0293-9

Fu, R., Song, Y., \& Zhao, W. (2014). Human Activity Recognition with Smartphones. Academic Press.

Guyon, I., \& Elisseeff, A. (2006). An introduction to feature extraction. In Feature extraction (pp. 1-25). Springer. doi:10.1007/978-3-540-35488-8_1

Hea, Z., \& Jin, L. (2009). Activity recognition from acceleration data based on discrete cosine transform and SVM. In IEEE International Conference on Systems, Man and Cybernetics, SMC 2009, (pp. 5041-5044). IEEE. doi:10.1109/ICSMC.2009.5346042

He, Z.-Y., \& Jin, L.-W. (2008). Activity recognition from acceleration data using a model representation and SVM. International Conference on Machine Learning and Cybernetics, 4, 2245-2250.

Helbostad, J. L., Vereijken, B., Becker, C., Todd, C., Taraldsen, K., Pijnappels, M., \& Mellone, S. (2017). Mobile health applications to promote active and healthy ageing. Sensors (Basel), 17(3), 622. doi:10.3390/s17030622 PMID:28335475 
Huang, Y. M., \& Du, S. X. (2005). Weighted support vector machine for classification with uneven training class sizes. Proceedings of the IEEE international conference on machine learning and cybernetics, 7, 4365-4369.

Hsu, C. W., Chang, C. C., \& Lin, C. J. (2003). A practical guide to support vector classification. Academic Press.

Chang \& Lin. (2011). LIBSVM: A library for support vector machines. ACM Transactions on Intelligent Systems and Technology, 2, 27:1-27:27. Available at http://www.csie.ntu. edu.tw/_cjlin/libsvm

Khan, A. M., Siddiqi, M. H., \& Lee, S. (2013). Exploratory data analysis of acceleration signals to select light-weight and accurate features for real-time activity recognition on smartphones. Sensors (Basel), 13(10), 13099-13122. doi:10.3390/s131013099 PMID:24084108

Kose, M., Incel, O. D., \& Ersoy, C. (2012). Online human activity recognition on smart phones. Workshop on Mobile Sensing: From Smartphones and Wearables to Big Data, 16, 11-15.

Kwapisz, J. R., Weiss, G. M., \& Moore, S. A. (2011). Activity recognition using cell phone accelerometers. SIGKDD Explorations, 12(2), 74-82. doi:10.1145/1964897.1964918

Lara, O.D., \& Labrador, M.A. (2013). A Survey on Human Activity Recognition Using Wearable Sensors. IEEE Communications Surveys and Tutorials, 15(3), 1192-1209.

Liang, Y., Zhou, X., Guo, B., \& Yu, Z. (2018). Activity recognition using ubiquitous sensors: An overview. Wearable Technologies: Concepts, Methodologies, Tools, and Applications, 199-230.

Lester, J., Choudhury, T., \& Borriello, G. (2006). A Practical Approach to Recognizing Physical Activities. Proceedings of the 4th International Conference on Pervasive Computing, 1-16. doi:10.1007/11748625_1

Manirabona, A., Fourati, L. C., \& Boudjit, S. (2018). Investigation on healthcare monitoring systems: Innovative services and applications. In Wearable Technologies: Concepts, Methodologies, Tools, and Applications (pp. 1264-1283). IGI Global.

Menhour, I., Abidine, M. B., \& Fergani, B. (2018). A New Framework Using PCA, LDA and KNN-SVM to Activity Recognition Based SmartPhone's Sensors. In 2018 6th International Conference on Multimedia Computing and Systems (ICMCS) (pp. 1-5). IEEE.

Nguyen, N., \& Nguyen, D. (2015). Hidden markov model for stock selection. Risks, 3(4), 455-473. doi:10.3390/ risks3040455

Nimkar, S., \& Gilles, E. E. (2019). Improving Global Health With Smartphone Technology: A Decade in Review of mHealth Initiatives. Multigenerational Online Behavior and Media Use: Concepts, Methodologies, Tools, and Applications. International Journal of E-Health and Medical Communications, 9(3), 54-75.

Ordóñez, F. J., Duque, A., de Toledo, P., \& Sanchis, A. (2012). A hybrid HMM/ANN model for activity recognition in the home using binary sensors. Ambient Assisted Living and Home Care - $4^{\text {th }}$ International Workshop, WAAL 2012, 98-105.

Ordonez, F., de Toledo, J. P., \& Sanchis, A. (2013). Activity recognition using hybrid generative/discriminative models on home environments using binary sensors. Sensors (Basel), 13(5), 5460-5477. doi:10.3390/s130505460 PMID:23615583

Osuna, E., Freund, R., \& Girosi, F. (1997). Support vector machines: training and applications. Massachusetts Institute of Technology.

Rabiner, L. R. (1989, February). A tutorial on hidden Markov models and selected applications in speech recognition. Proceedings of the IEEE, 77(2), 257-286. doi:10.1109/5.18626

Reiss, A., Hendeby, G., \& Stricker, D. (2013). A competitive approach for human activity recognition on smartphones. European Symposium on Artificial Neural Networks, Computational Intelligence and Machine Learning (ESANN 2013), 455-460.

Ronao, C. A., \& Cho, S. B. (2014). Human activity recognition using smartphone sensors with two-stage continuous hidden Markov models. International Conference on Natural Computation.

Peterek, T., Penhaker, M., Gajdoš, P., \& Dohnálek, P. (2014). Comparison of classification algorithms for physical activity recognition. Innovations in Bio-inspired Computing and Applications. doi:10.1109/ICNC.2014.6975918 
Shoaib, M. (2013). Human Activity Recognition Using Heterogeneous Sensors,. 2013 ACM Conference on Ubiquitous Computing, UbiComp'13 Adjunct.

Shoaib, M., Bosch, S., Incel, O. D., Scholten, H., \& Havinga, P. J. M. (2014). Fusion of Smartphone Motion Sensors for Physical Activity Recognition. Sensors (Basel), 14(6), 10146-10176. doi:10.3390/s140610146 PMID:24919015

Shoaib, M., Bosch, S., Incel, D., Scholten, H., \& Havinga, P. J. M. (2015). A Survey of Online Activity Recognition Using Mobile Phones. Journal of Sensors (Basel, Switzerland), 15(1), 2059-2085. doi:10.3390/ s150102059 PMID:25608213

Siirtola, P., \& Roning, J. (2012). Recognizing Human Activities User independently on Smartphones Based on Accelerometer Data. Special Issue on Distributed Computing and Artificial Intelligence, 1(5), 38-45. doi:10.9781/ijimai.2012.155

Su, X., Tong, H., \& Ji, P. (2014). Activity recognition with smartphone sensors. Tsinghua Science and Technology, 19(3), 235-249. doi:10.1109/TST.2014.6838194

Tamura, T., Sekine, M., Ogawa, M., Togawa, T., \& Fukui, Y. (1997). Classification of acceleration wave forms during walking by wavelet transform. Methods of Information in Medicine, 36(45), 356-359. PMID:9470398

Tian, J., Gu, H., \& Liu, W. (2011). Imbalanced classification using support vector machine ensemble. Neural Computing \& Applications, 20(2), 203-209. doi:10.1007/s00521-010-0349-9

Van Kasteren, T., Englebienne, G., \& Kröse, B. J. (2010). An activity monitoring system for elderly care using generative and discriminative models. Personal and Ubiquitous Computing, 14(6), 489-498. doi:10.1007/ s00779-009-0277-9

Walse, K. H., Dharaskar, R. V., \& Thakare, V. M. PCA Based Optimal ANN Classifiers for Human Activity Recognition Using Mobile Sensors Data. In Proceedings of First International Conference on Information and Communication Technology for Intelligent Systems: Volume 1 (pp. 429-436). Springer International Publishing, 2016. doi:10.1007/978-3-319-30933-0_43

Wu, G. D., \& Huang, P. H. (2010, April). A Maximizing-Discriminability-Based Self-Organizing Fuzzy Network for Classification Problems. IEEE Transactions on Fuzzy Systems, 18(2). doi:10.1109/TFUZZ.2010.2098879

Yan, G. T., Ma, G. F., \& Xiao, Y. Z. (2007). Support vector machines based on hybrid kernel function. Journal of Harbin Institute of Technology, 39(11), 1704-1706.

Yan, Z., Subbaraju, V., Chakraborty, D., Misra, A., \& Aberer, K. (2012). Energy-efficient continuous activity recognition on mobile phones: An activity-adaptive approach. Wearable Computers (ISWC), 16th International Symposium on, 17-24.

Zainudin, M. S., Mohamed, R., Sulaiman, M. N., Perumal, T., Mustapha, N., \& Nazri, A. S. A. (2017). MultiLabel Classification Using Label Combination To Recognize Human Activity Based On Various Sensor Positions. Proceedings of the 6th International Conference on Computing and Informatics, ICOCI.

Zheng, T., Du, Z. J., Sun, L., Huo, M., \& Chen, Y. (2018). TASG: An Augmented Classification Method for Impersonal HAR. Mobile Information Systems. 